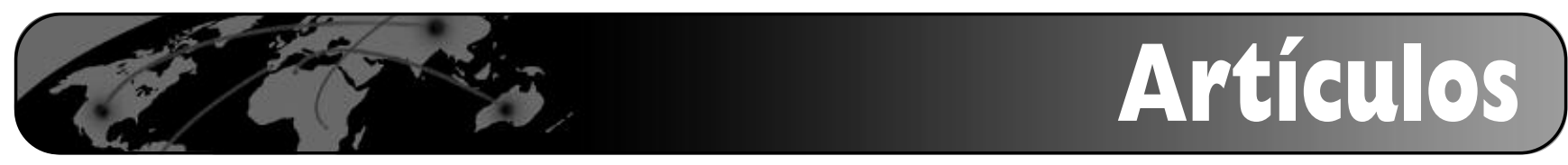

\title{
La vigilancia tecnológica en la gestión de proyectos de I+D+i: recursos y herramientas
}

\author{
Por Javier Muñoz Durán, María Marín Martínez y José Vallejo Triano
}

\begin{abstract}
Resumen: La vigilancia tecnológica se ha convertido en básica para las organizaciones que gestionan proyectos de I+D+i. La publicación de la norma UNE 166006:2006 EXGestión de la I+D+i: sistemas de vigilancia tecnológica, sistematiza la implantación de esta actividad dentro de las organizaciones innovadoras. Exponemos y describimos los procesos fundamentales para desarrollar eficientemente esta actividad por parte de los profesionales de la gestión de la información. A su vez, enumeramos herramientas y recursos útiles, que servirán de ayuda para la búsqueda, recuperación y posterior tratamiento de la información, y dotarán a ésta del valor añadido necesario para la toma de decisiones, al igual que ofrecemos una reflexión sobre las patentes, como principal fuente estructurada de información tecnológica.
\end{abstract}

Palabras clave: Vigilancia tecnológica, Gestión, Investigación, Desarrollo, Innovación, Patentes, UNE 166006, Gestión $I+D+i$.

\section{Title: Technological surveillance in the man- agement of $R \& D$ and innovation projects: re- sources and tools}

Abstract: Technological surveillance systems have become a fundamental tool for organisations that manage $R \& D$ and innovation projects. The publication of the UNE 166006:2006 EX standard-Management of $R \& D$ and innovation: technological surveillance systems systematises the manner in which this activity is set up within innovation organisations. We present and describe the main processes required for the efficient development of this activity by information management professionals. We also list some useful resources and tools that will help in the search, recovery and subsequent handling of information, by which the value of the retrieved information is enhanced for supporting the decision making process. Additionally, we comment on patents and their importance as a major structured source of technological information.

Keywords: Technological surveillance, Management, Research, Development, Innovation, Patents, UNE 166006, R\&D.

Muñoz Durán, Javier; Marín Martínez, María; Vallejo Triano, José. “La vigilancia tecnológica en la gestión de proyectos de I+D+i: recursos y herramientas”. En: El profesional de la información, 2006, noviembre-diciembre, v. 15, n. 6 , pp. 411-419.

\section{Introducción}

La vigilancia tecnológica (VT) dentro de las organizaciones innovadoras es una función fundamental para poder gestionar proyectos de $\mathrm{I}+\mathrm{D}+\mathrm{i}$, siendo las normas de la serie 166000: Gestión de la $I+D+i$ básicas para llevar a cabo con éxito este tipo de proyectos. En mayo de 2006 se publicó la norma UNE: 166006 EX-Gestión de la $I+D+i$ : sistema de vigilancia tecnológica, que sistematiza esta actividad. 


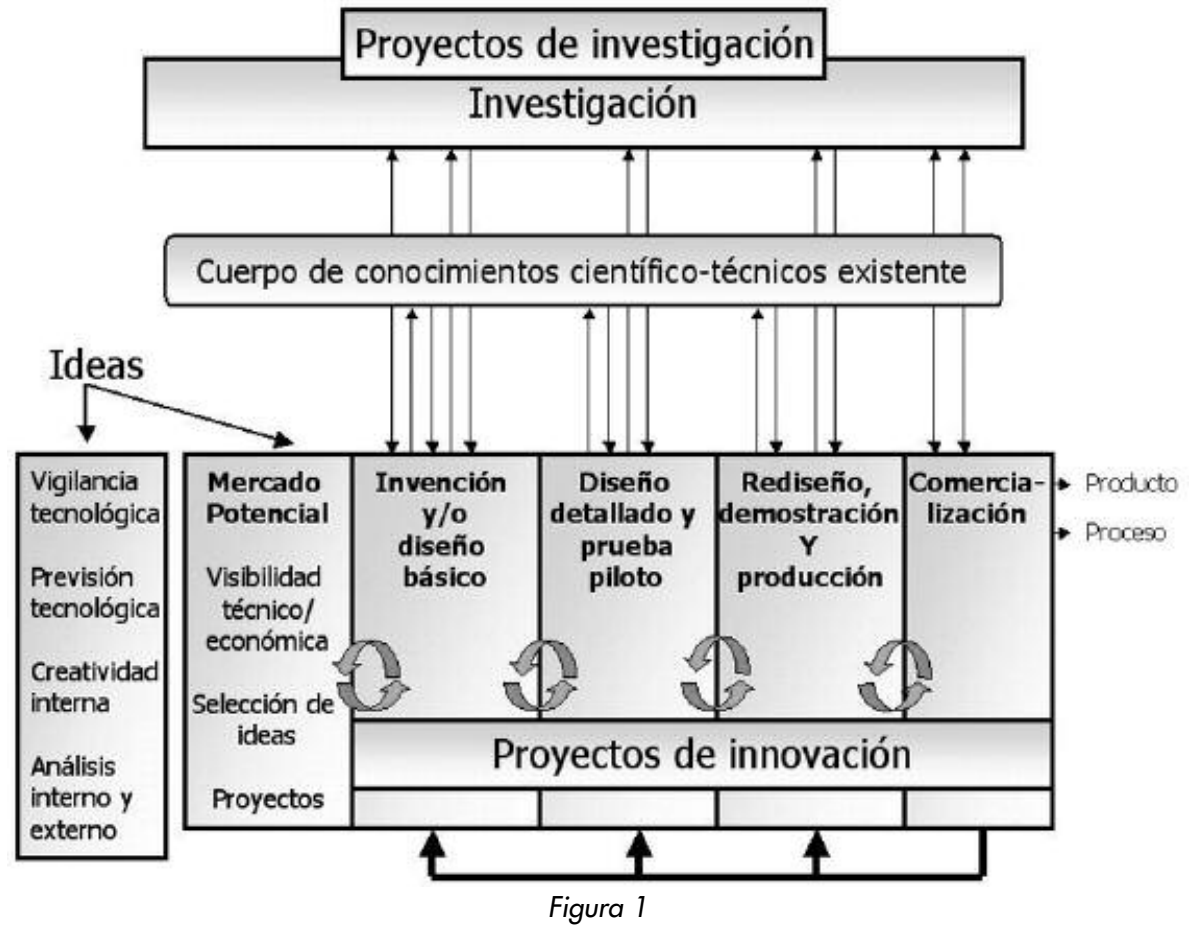

El objetivo que perseguimos en este trabajo es describir, en base a esta norma, las diferentes etapas necesarias para implantar un sistema de VT en cualquier organización que gestione proyectos de I+D+i. Presentamos los procesos involucrados: establecimiento de objetivos, selección de fuentes, búsqueda, tratamiento y divulgación de la información, complementando cada uno con útiles que ilustran los conceptos.

Resaltamos la vigilancia de las patentes como fuente de información tecnológica por ser un recurso de información estructurado y por la exclusividad de sus contenidos ya que se estima que más del $80 \%$ del conocimiento técnico actual está contenido en la documentación de patentes (Callon, et al. 1993).

\section{La vigilancia en la gestión de la I+D+i}

El análisis de la información que nos rodea es pieza fundamental en el proceso de innovación tecnológica. En el curso de los proyectos de I+D a menudo se ignora que las soluciones a los problemas técnicos que se presentan, o incluso el desarrollo que se persigue, están ya disponibles en el cuerpo de conocimientos científico-técnicos existente, formado por los artículos técnicos, las patentes y las ponencias en los congresos. Muchas veces no se vigila lo suficiente y cuando una organización consigue elaborar un producto se encuentra que éste ya está patentado.

¿Cómo se integra la vigilancia en la I+D+i? Para responder a esta pregunta debemos indicar que el proceso innovador se realiza mediante interacciones, y que el modelo que lo refleja aparece en la norma $U N E$ 166002 .
Su punto de partida es el mercado potencial configurado a partir de la la VT. Con su ayuda se identifica una serie de ideas para satisfacer nuevas necesidades de mercado o mejora de productos y/o procesos ya existentes. El elemento central es la idea y si la podemos llevar con éxito al mercado podremos hablar de innovación. Gracias a la VT obtendremos información para:

-Aplicar nuevas tecnologías.

- Crear nuevos productos.

-Evaluar el posible impacto de un hecho o cambio en el entorno.

La tarea no es fácil ya que la mayor parte de la información necesaria para captar nuevas ideas se encuentra en el exterior de las organizaciones, siendo además compleja y abundante. Organizarla y tratarla para añadirle valor, sirva para la toma de decisiones y se pueda disponer de ella en el momento oportuno, son cometidos de la vigilancia necesarios para la innovación. La vigilancia, por tanto, contribuye dentro del proceso innovador a reducir las decisiones erróneas en el inicio de un proyecto de investigación y desarrollo, así como en las fases de lanzamiento previo al mercado.

La norma UNE: 166002 define la VT como: "una forma organizada, selectiva y permanente de captar información del exterior sobre tecnología, analizarla y convertirla en conocimiento para tomar decisiones con menor riesgo y poder anticiparse a los cambios".

Hasta aquí parece clara la necesidad de practicar la vigilancia en toda organización que se considere innovadora. El siguiente paso es implantar el sistema. 


\section{Cómo organizar la vigilancia}

Una forma de organizar la VT, y que nos ayude a integrarla dentro de un sistema de gestión es mediante procesos, los cuales, según la norma ISO 9000, son "un conjunto de actividades mutuamente relacionadas o que interactúan, las cuales transforman elementos de entrada en resultados". Así, los procesos involucrados en la VT tienen como objetivo la obtención de información del entorno tecnológico, información que una vez tratada servirá para la toma de decisiones dentro de la organización. Una representación esquemática del proceso de VT puede verse en la figura 2.

\section{Identificación de necesidades}

Las necesidades de información serán determinadas por la organización en base a un autodiagnóstico que deberá tener en cuenta tanto la situación tecnológica de partida como las señales que recibe de su entorno: proveedores, clientes, competidores, etc.

De esta forma obtendremos los "factores críticos de vigilancia" (FCVs) que serán los que indiquen el tipo de información a buscar. Son cuestiones externas a la organización, cuya evolución afecta de modo crítico a la competitividad de la misma. Por ejemplo, con respecto al departamento de compras, un FCV puede ser la existencia de un nuevo proveedor, lo que puede suponer un sustancial ahorro de costes de fabricación. Una vez detectados, y dependiendo del área en donde se encuadren los FCVs, así será la VT a realizar, que podrá ser de los siguientes tipos:

-De las tecnologías disponibles o que acaban de aparecer, capaces de intervenir en nuevos productos o procesos.

-Competitiva: se ocupa de la búsqueda de información sobre los competidores actuales y los potenciales.

-Comercial: estudia los datos referentes a productos, mercados, clientes y proveedores (estudios de mercado, evolución de las necesidades de los clientes y de su solvencia, nuevos productos ofrecidos por los proveedores, etc.).

-Del entorno: abarca la detección de aquellos hechos exteriores que pueden condicionar el futuro en áreas como la sociología, la política, el medio ambiente, las reglamentaciones, etc.

\section{Identificación de fuentes}

Una vez se tienen claras las necesidades debemos identificar qué recursos son los que nos proporcionan la información relevante para controlar los FCVs. Éste no es un paso sencillo.

Hace unos años, antes de la revolución de las tecnologías de la información y de la comunicación, tener controlados los avances tecnológicos era relativamente sencillo. El tamaño de la comunidad científica era menor, las innovaciones se producían en unos pocos países y el número de publicaciones especializadas era bastante reducido. En la actualidad nos movemos en un mundo de excesiva información, cada año se publican unos dos millones de artículos y se registra un millón de patentes. Nos encontramos entonces ante un entorno en donde la información es mayor que nunca y gran parte de ella accesible libremente. Debido a ese gran volumen de datos, la dificultad no está en encontrar la información, sino en saberla gestionar y extraer de ella ventajas desde el punto de vista de la competitividad de nuestra organización.

Las fuentes de información son múltiples y no todas son igual de eficaces para los distintos tipos de vigilancia a los que antes hicimos referencia. Es necesario, por tanto, clasificar las fuentes (figura 3).

Las informales se caracterizan porque su origen suele ser las conversaciones o negociaciones con clientes, proveedores, investigadores, etc. Muchas veces son de alto valor al tener una relación directa con la línea de trabajo de la organización y pueden mostrar posibles estrategias o futuras investigaciones de los

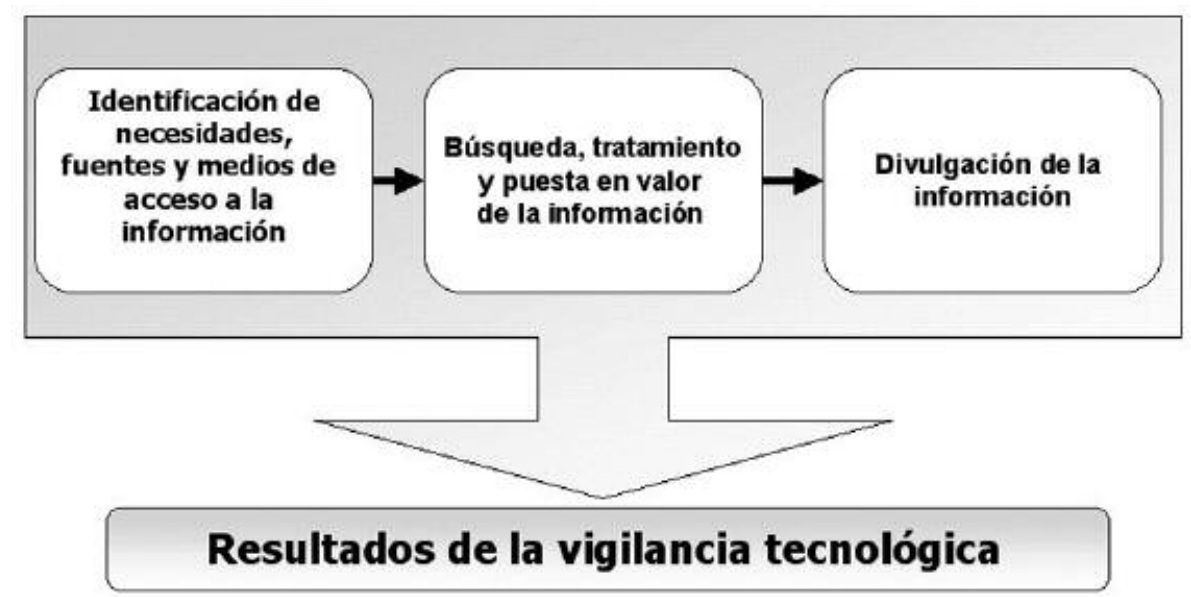

Figura 2 


\begin{tabular}{|l|l|}
\hline Fuentes de información \\
\hline Formales & Informales \\
\hline Prensa & Notas o apuntes \\
\hline Patentes & Conversaciones \\
\hline Bases de datos & $\begin{array}{l}\text { Visitas a ferias, exposiciones, } \\
\text { salones }\end{array}$ \\
\hline $\begin{array}{l}\text { Publicaciones de otras } \\
\text { empresas }\end{array}$ & $\begin{array}{l}\text { Congresos, seminarios, } \\
\text { jornadas }\end{array}$ \\
\hline $\begin{array}{l}\text { Publicaciones de organismos } \\
\text { oficiales }\end{array}$ & Encuestas no oficiales \\
\hline Libros & Esquemas o bocetos \\
\hline
\end{tabular}

Figura 3

competidores. El inconveniente que presentan es que hay que estructurarlas para poder archivarlas y recurrir a ellas cuando se necesiten.

Las formales son bases de datos o servicios de internet. Su interés radica en que tienen homogenizado el formato de las informaciones y ofrecen la posibilidad de automatizar su explotación. Cuanto más estructurada esté su información más sencillo será realizar posteriores tratamientos estadísticos. El problema que presentan es que necesitan un tiempo para afinar las estrategias de búsqueda, así como para comparar las informaciones que se van descargando de las bases de datos. Aquí es donde juega un papel fundamental el profesional de la información que será el que fije esas

\begin{tabular}{|l|l|}
\hline \multicolumn{2}{|l|}{ Noticias y datos de mercado } \\
\hline Informes de países \\
\hline CIA & $\begin{array}{l}\text { http://www.odci.gov/cia/public } \\
\text { ations/factbook/index.htmI }\end{array}$ \\
\hline ICEX & http://www.icex.es \\
\hline Estudios de mercado \\
\hline NTDB (EUA)* & $\begin{array}{l}\text { http://www.stat- } \\
\text { usa.gov }\end{array}$ \\
\hline $\begin{array}{l}\text { Strategis } \\
\text { (Canadá) }\end{array}$ & $\begin{array}{l}\text { http://www.strategis } \\
\text { ic.gc.ca/engdoc/ } \\
\text { search.html }\end{array}$ \\
\hline Jetro (Japón) & $\begin{array}{l}\text { http://www.jetro.go. } \\
\text { jp/ec/e/market/ } \\
\text { index.html }\end{array}$ \\
\hline $\begin{array}{l}\text { Frost \& Sullivan } \\
\text { (Europa)* }\end{array}$ & $\begin{array}{l}\text { http://www.frost. } \\
\text { com }\end{array}$ \\
\hline DBK (España)* & http://www.dbk.es \\
\hline Bases de datos de mercados \\
\hline Profound* & $\begin{array}{l}\text { http://www.profound. } \\
\text { co.uk }\end{array}$ \\
\hline Dialog* & $\begin{array}{l}\text { http://www.dialogweb. } \\
\text { com } \\
\text { http://www.dialogclassic. } \\
\text { com }\end{array}$ \\
\hline Data-Star* & http://datastarweb.com \\
\hline TradStat* & http://tradstatweb.com \\
\hline (*) De pago & Figura 4 \\
\hline
\end{tabular}

Figura 4 estrategias y ecuaciones de búsqueda para tratar la información adquirida.

\section{Medios de acceso}

En las figuras de la 4 a la 9 comentamos diversas herramientas, disponibles en internet, con las cuales podremos obtener información de las fuentes que antes hemos mencionado e incluso algunas de ellas permitirán la automatización de tareas. Los medios de acceso son muy heterogéneos y continuamente están apareciendo nuevos servicios que ofrecen información de muy diversa índole, algunos son gratuitos y otros no. Por tanto, es una decisión a tomar dentro de la or-

\begin{tabular}{|c|c|}
\hline \multicolumn{2}{|c|}{ Vigilancia de medios de prensa } \\
\hline Iconoce & $\begin{array}{l}\text { http://www. iconoce.com } \\
\text { Escanea unos } 500 \text { medios, } \\
\text { recopila } 1.500 \text { noticias/día. } \\
\text { Permite búsqueda a texto } \\
\text { completo. } \\
\text { Servicio de alertas. }\end{array}$ \\
\hline iMente & $\begin{array}{l}\text { http://www.imente.com } \\
\text { Recopila titulares de } 13.000 \\
\text { fuentes en } 13 \text { idiomas, } 55.000 \\
\text { noticias/día. } \\
\text { Permite búsqueda en título y } \\
\text { texto. } \\
\text { Servicio de alertas. }\end{array}$ \\
\hline Acceso & $\begin{array}{l}\text { http://www.acceso.com } \\
\text { Recopila selectivamente } \\
\text { diferentes tipos de: } \\
\text { Notas de prensa. } \\
\text { Eventos o cursos que organiza } \\
\text { una empresa. } \\
\text { Comunicados financieros. } \\
\text { Teletipos. }\end{array}$ \\
\hline $\begin{array}{l}\text { My } \\
\text { News }\end{array}$ & $\begin{array}{l}\text { http://www. mynews.es/ } \\
\text { Texto completo de más de } 100 \\
\text { periódicos, mayoritariamente } \\
\text { españoles. } \\
\text { Servicio de alerta. } \\
\text { Consulta online de hemeroteca. }\end{array}$ \\
\hline \multicolumn{2}{|c|}{ Todos son de pago } \\
\hline
\end{tabular}




\begin{tabular}{|l|l|}
\hline \multicolumn{2}{|c|}{$\begin{array}{r}\text { Vigilancia de empresas españolas e } \\
\text { internacionales }\end{array}$} \\
\hline Ardan & $\begin{array}{l}\text { http://www.ardan.es } \\
\text { Informes comerciales. } \\
\text { Análisis sobre la competencia. }\end{array}$ \\
\hline Informa & $\begin{array}{l}\text { http://www.informa.es } \\
\text { Datos e informes comerciales. } \\
\text { Análisis de balances. }\end{array}$ \\
\hline e-Informa & $\begin{array}{l}\text { http://www.e-informa.com } \\
\text { Datos e informes comerciales, } \\
\text { sectoriales, ayudas }\end{array}$ \\
\hline Dun\&Bradstreet & $\begin{array}{l}\text { http://www.dnb.com } \\
\text { Informes comerciales. } \\
\text { Informes de crédito. } \\
\text { Alertas con incidencias. }\end{array}$ \\
\hline $\begin{array}{l}\text { Dialtp://login.profiles.dialog.com/ } \\
\text { dialog } \\
\text { Profiles }\end{array}$ & $\begin{array}{l}\text { Recopilación de información de } \\
\text { empresas de otras bases de } \\
\text { datos }\end{array}$ \\
\hline Todos son de pago \\
\hline
\end{tabular}

\begin{tabular}{|l|l|}
\hline \multicolumn{3}{|l|}{ Monitorización de páginas web } \\
\hline Karnak & $\begin{array}{l}\text { Envía una alerta con el } \\
\text { número de nuevos resultados } \\
\text { en un tema de interés }\end{array}$ \\
\hline Tracerlock & $\begin{array}{l}\text { http://www. tracerlock.com } \\
\text { Envía una alerta con los } \\
\text { nuevos resultados obtenidos } \\
\text { los cambios de una página } \\
\text { Web }\end{array}$ \\
\hline Northernlight & $\begin{array}{l}\text { http://www.northernlight.com } \\
\text { Envía un enlace avisando que } \\
\text { hay nuevos resultados sobre } \\
\text { un tema de interés }\end{array}$ \\
\hline Todos son de pago
\end{tabular}

Figura 7

Figura 6

\begin{tabular}{|l|l|}
\hline Monitorización de páginas web con agentes rastreadores \\
\hline WebsiteWatcher & $\begin{array}{l}\text { http://www.aignes.com } \\
\text { Permite: } \\
\text { Chequear un numero ilimitado de sitios web para detectar cambios. } \\
\text { Grabar macros para llegar a páginas web cuya dirección se ignora. } \\
\text { Utilizar expresiones regulares para definir los mecanismos de filtrado. } \\
\text { Chequear actualizaciones automáticamente a una velocidad de más } \\
\text { de 100 direcciones web por minuto. }\end{array}$ \\
\hline $\begin{array}{l}\text { Copernic Agent } \\
\text { Pro }\end{array}$ & $\begin{array}{l}\text { Meta motor de búsqueda capaz de filtrar los resultados con palabras } \\
\text { clave, clasificar por pertinencia, etc. } \\
\text { Puede enviar alertas con los nuevos resultados de una búsqueda o } \\
\text { con las modificaciones de una página } \\
\text { Se integra como barra de herramientas en IExplorer }\end{array}$ \\
\hline \multicolumn{2}{|l}{ Todos son de pago } \\
\hline
\end{tabular}

Figura 8

\section{Pluggins en el navegador (IExplorer)}

Se incorporan como una nueva barra de herramientas con nuevas funciones y utilidades. La principal ventaja es que evitan tener que ir a los sitios web de los buscadores a plantear la búsqueda.

\begin{tabular}{|l|l|} 
Vivisimo tool bar & $\begin{array}{l}\text { http://vivisimo.com/toolbar/toolbar-download.html } \\
\text { Es un meta motor que analiza el texto de los resultados, crea categorías } \\
\text { según los términos más representativos y agrupa los resultados en dichas } \\
\text { categorías }\end{array}$ \\
Copernic-Meta & $\begin{array}{l}\text { Integra la ventana de búsqueda en la barra de tareas. Es personalizable } \\
\text { para incluir todos los motores de búsqueda que se deseen }\end{array}$ \\
Scirus tool bar & $\begin{array}{l}\text { http://www.scirus.com/srsapp/toolbar/ } \\
\text { Permite lanzar búsquedas en varias secciones de Scirus o en el buscador } \\
\text { Al/theweb }\end{array}$ \\
\hline
\end{tabular}




\begin{tabular}{|l|l|}
\hline BrightPlanet & http://www.brightplanet.com \\
\hline Profusion & http://www.profusion.com \\
\hline CompletePlanet & http://www.completeplanet.com \\
\hline InternetInvisible & http://www.internetinvisible.com \\
\hline \multicolumn{2}{r}{ Figura 10}
\end{tabular}

ganización con respecto al sistema de VT el presupuesto que se va a destinar a esta actividad, ya que esto condicionará el tipo de fuentes a seleccionar.

Debemos comentar en este punto la existencia de una fuente formal denominada "web profunda" o "web invisible" formada por las bases de datos accesibles desde internet que son interrogables mediante formularios y que generan páginas dinámicas con cada pregunta. Tienen la particularidad de que los buscadores convencionales (Google, Yahoo, etc.) no pueden entrar en ellas. El estudio realizado por Michael K. Bergman (2000) arroja una serie de datos que nos pueden dar una idea del tamaño de la parte invisible de la Red: contiene cerca de 550 billones de documentos individuales, frente al billón de páginas de la "superficie". Esto supone que más de 200.000 sitios son invisibles, con cantidades de contenidos $1.000-2.000$ veces mayores que los de la web visible. Se muestran algunos ejemplos en la figura 10 .

3.1. Fuentes de información formal y estructurada: patentes y bases de datos documentales

Una de las principales fuentes de información para la VT la constituyen las patentes: es un título otorgado por el estado que concede un derecho de explotación exclusivo de una invención en todo el territorio del país o países en los que se registre por un período de 20 años.
¿Por qué es importante analizarlas? Desde el punto de vista de la información que contienen, representan una fuente de información absolutamente estratégica. En ellas se describen tecnologías y/o productos con aplicaciones muy concretas que a veces ni si quiera se han comercializado aún. Es importante destacar también que, mas del $80 \%$ de la información recogida en ellas no se publica en ningún otro medio. Para organizaciones que se dediquen a la $\mathrm{I}+\mathrm{D}+\mathrm{i}$ el análisis de las patentes les será muy útil ya que les permite:

- Ver la línea de desarrollos de la competencia.

-Identificar nuevos competidores potenciales.

—Establecer y comparar ofertas tecnológicas.

-Relacionar competidor-tecnología-mercado.

Desde el punto de vista de cómo se presenta la información, los documentos de las patentes están normalizados y se estructuran en las siguientes partes:

-Portada/primera hoja: es el resumen del contenido de todo el documento y además incluye los siguientes datos: título, inventor, patentador, fechas y números de prioridad, solicitud y publicación, clasificaciones, patentes citadas y gráfico más representativo. También se incluye un breve resumen que describe el problema técnico que se ha solucionado.

- La memoria descriptiva.

-Las reivindicaciones.

—Los gráficos.

Algunas aplicaciones para la utilización de las patentes como fuente de información son las presentadas en las figuras 11 y 12. Otros recursos importantes de

\begin{tabular}{|l|l|}
\hline \multicolumn{2}{|l|}{ Productores de bases de datos } \\
\hline Delphion* & $\begin{array}{l}\text { http://www.delphion.com } \\
\text { Da acceso a distintas colecciones de patentes: PCT, EP y US a texto completo } \\
\text { Descarga/venta de documentos, grabación de búsquedas, alertas, etc. } \\
\text { Software para gestión de la propiedad industrial }\end{array}$ \\
\hline Derwent* & $\begin{array}{l}\text { http://www.derwent.com } \\
\text { Propiedad de Thomson. Produce un conjunto de bases de datos de patentes, de alto } \\
\text { valor añadido. La más famosa es World Patent Index. } \\
\text { Cobertura de más de } 40 \text { países. } \\
\text { Clasificaciones propias para química y electricidad. }\end{array}$ \\
\hline Micropatent* $*$ & $\begin{array}{l}\text { http://www.micropatent.com } \\
\text { Texto completo: USA, EP, PCT, GB, DE. } \\
\text { Tecnología de mapas Aurigin. }\end{array}$ \\
\hline Espacenet & $\begin{array}{l}\text { http://es.espacenet.com } \\
\text { Fondo documental de la oficina Europea de Patentes. } \\
\text { Enlaces a 30 millones de documentos. } \\
\text { Integra datos del estado legal de cada patente de la base de datos Inpadoc. }\end{array}$ \\
\hline (*) De pago & \multicolumn{2}{l}{} \\
\hline
\end{tabular}




\begin{tabular}{|l|l|}
\hline \multicolumn{1}{|c|}{ Distribuidores de bases de datos } \\
\hline Dialog & $\begin{array}{l}\text { http://www.dialogselect.com } \\
\text { http://www.dialogclassic.com } \\
\text { Reúne un gran número de bases } \\
\text { de datos. } \\
\text { Descarga/venta de documentos, } \\
\text { grabar búsquedas, alertas... } \\
\text { Software para gestión de la } \\
\text { propiedad industrial. }\end{array}$ \\
\hline Questel \\
$\begin{array}{l}\text { http://www.questel.orbit.com/ } \\
\text { index.htm }\end{array}$ \\
$\begin{array}{l}\text { Permite búsqueda por estructuras } \\
\text { químicas. } \\
\text { Especializado en propiedad } \\
\text { industrial. }\end{array}$ \\
$\begin{array}{l}\text { http://www.cas.org/stn.html } \\
\text { CAS (Chemical Abstracts Service): } \\
\text { Especializada en química-farmacia, } \\
\text { incluye patentes. } \\
\text { Búsqueda por estructuras } \\
\text { químicas. }\end{array}$ \\
\hline Todos de pago \\
\hline pTN
\end{tabular}

Figura 12

\begin{tabular}{|l|l|}
\hline Base de datos & Temática \\
\hline $\begin{array}{l}\text { Science citation } \\
\text { index (SCI) }\end{array}$ & Ciencia y tecnología \\
\hline $\begin{array}{l}\text { Chemical } \\
\text { abstracts }\end{array}$ & Química \\
\hline Medline & Medicina \\
\hline Compendex & Ingeniería \\
\hline Inspec & Electricidad y electrónica \\
\hline Biosis & Ciencias de la vida \\
\hline Cindoc/CSIC & Ciencia y tecnología \\
\hline ABI-Inform & $\begin{array}{l}\text { Gestión empresarial. } \\
\text { Divulgación tecnológica. }\end{array}$ \\
\hline $\begin{array}{l}\text { Social science } \\
\text { citation index }\end{array}$ & Ciencias sociales \\
\hline
\end{tabular}

Figura 13

información formal estructurada son las bases de datos documentales, destacando entre ellas las de la figura 13.

\section{Búsqueda}

Identificadas las necesidades y las fuentes de información, debemos comenzar la búsqueda. Ésta es un proceso iterativo en donde siempre habrá que analizar los resultados para chequear si éstos se corresponden con lo esperado, y si no es así volver a iniciarlo. La búsqueda y selección de la información se realizará estableciendo una estrategia de búsqueda previa en las fuentes seleccionadas, que deberá incluir:

- Listado de los términos controlados, clasificados y agrupados según distintos puntos de vista.
-Especificación de los conceptos de mayor y menor interés.

-Identificación de las fuentes pertinentes.

Como resultado obtendremos informaciones formales que podremos complementar con otras de carácter informal.

\section{Puesta en valor de la información}

Una vez validada la información obtenida, debemos analizarla para darle valor de cara a la toma de decisiones. Este análisis dependerá del volumen de información a analizar (manejable o no), del contenido o su naturaleza (mediciones, artículos, datos de encuestas) así como de su formato y estructura (informales, escritas, formalizadas).

Centrándonos de nuevo en la patentes por las razones que anteriormente comentamos, gracias a su estructuración podemos hacer múltiples análisis estadísticos que nos ayudarán a extraer nuevos datos a parte de los ya contenidos en sus documentos por separado. Cruzando diferentes campos de las fichas de las patentes podemos dar respuesta a las cuestiones propuestas en la figura 14. Para realizar este tipo de análisis estadístico existen programas que trabajan directamente con las patentes sin tener que modificarlas (figura 15).

\begin{tabular}{|l|l|}
\hline Pregunta & Tipo de cruce de datos \\
\hline $\begin{array}{l}\text { ¿Con qué empresas } \\
\text { cooperan mis } \\
\text { competidores? }\end{array}$ & Análisis de patentadores \\
\hline $\begin{array}{l}\text { ¿Cuál es el principal } \\
\text { esfuerzo investigador } \\
\text { de la competencia? }\end{array}$ & $\begin{array}{l}\text { Análisis de empresas } \\
\text { según la IPC (Intl. patent } \\
\text { classification) }\end{array}$ \\
\hline $\begin{array}{l}\text { ¿Desde cuándo } \\
\text { investigan mis } \\
\text { competidores en este } \\
\text { campo? }\end{array}$ & $\begin{array}{l}\text { Análisis de patentadores } \\
\text { /fechas }\end{array}$ \\
\hline $\begin{array}{l}\text { ¿Qué técnicas se } \\
\text { están transfiriendo a } \\
\text { nuevas áreas o } \\
\text { productos? }\end{array}$ & Análisis IPC /IPC \\
\hline $\begin{array}{l}\text { ¿Qué técnicas están } \\
\text { emergiendo y cuáles } \\
\text { están en declive? ¿En } \\
\text { qué estado del ciclo } \\
\text { está una tecnología? }\end{array}$ & Análisis IPC /año \\
\hline $\begin{array}{l}\text { ¿Cuáles son los países } \\
\text { líderes en cierta } \\
\text { tecnología? }\end{array}$ & Análisis de prioridades \\
\hline $\begin{array}{l}\text { ¿Cuáles son los } \\
\text { principales mercados } \\
\text { para una tecnología? }\end{array}$ & Análisis de países destino \\
\hline $\begin{array}{l}\text { ¿Cómo está } \\
\text { evolucionando la I+D } \\
\text { de mis competidores? }\end{array}$ & Análisis IPC /fechas \\
\hline $\begin{array}{l}\text { ¿Quiénes son los } \\
\text { principales expertos } \\
\text { en esta tecnología? }\end{array}$ & $\begin{array}{l}\text { Análisis de inventores } \\
\text { /IPC }\end{array}$ \\
\hline
\end{tabular}




\begin{tabular}{|c|c|}
\hline \multicolumn{2}{|c|}{ Software para el análisis estadístico de patentes } \\
\hline $\begin{array}{l}\text { Delphion } \\
\text { PatentLabII }\end{array}$ & $\begin{array}{l}\text { http://www.delphion.com/products/research/products-patlab } \\
\text { Puede descargar información de Delphion y hacer diversos análisis de las patentes } \\
\text { creando gráficos de } 2 \text { ó } 3 \text { dimensiones, mediante la combinación de los campos que } \\
\text { se desee analizar. } \\
\text { Patenlab identifica y analiza tendencias, áreas clave y nuevos desarrollos, así como } \\
\text { potenciales de productos de los competidores y permite descubrir relaciones no } \\
\text { evidentes en grandes conjuntos de patentes. } \\
\text { El software es gratis pero hay que pagar la descarga de información. }\end{array}$ \\
\hline $\begin{array}{l}\text { Matheo } \\
\text { Patent }\end{array}$ & $\begin{array}{l}\text { http://www. matheo-software.com } \\
\text { Extracción automática de patentes de las bases de datos Espacenet y Uspto. } \\
\text { Análisis estadísticos y bibliométricos combinando dos campos de información. } \\
\text { Creación de matrices, gráficos y redes utilizando campos específicos de las patentes. } \\
\text { Especializado en propiedad industrial. } \\
\text { Licencia: } 600 \text { /año. }\end{array}$ \\
\hline $\begin{array}{l}\text { Derwent } \\
\text { Analytics }\end{array}$ & $\begin{array}{l}\text { http://thomsonderwent.com/products/dapt/derwentanalytics } \\
\text { Software de minería de datos y de visualización de grandes volúmenes de } \\
\text { información de patentes obtenidas de las bases de datos Thomson Derwent, } \\
\text { especialmente WPI. } \\
\text { Se extraen enfoques críticos para poder tomar decisiones de negocio. } \\
\text { Licencia: } 19.000 \text { US } \$ 10.000 \text { referencias }\end{array}$ \\
\hline & \\
\hline
\end{tabular}

Figura 15

Otras técnicas más avanzadas para el análisis de la información permiten trabajar no ya con campos predefinidos (autor, fecha publicación, etc.) sino con los datos individuales contenidos en esos campos (palabras del resumen, apellidos del autor, etc.). Estamos hablando de la minería de datos que se aplica a fuentes de información estructurada (bases de datos de patentes y documentales). Las operaciones básicas de análisis que podemos hacer son: clasificación, recuento y cruces o coocurrencias. El recuento de términos puede servir para detectar, por ejemplo, la existencia de tecnologías emergentes. Con la coocurrencia o análisis de la aparición conjunta de palabras, se podría identificar áreas de estudio en donde dichas términos intervienen. Algunos posibles análisis de concurrencias de ejemplo las incluimos en la figura 16 , mientras que en la 17 proponemos varias herramientas con las que poder realizar minería de datos.

\begin{tabular}{|l|l|}
\hline Coocurrencias & Resultado del análisis \\
\hline Entre empresas & Redes de colaboración \\
\hline $\begin{array}{l}\text { Entre empresas y } \\
\text { fechas }\end{array}$ & $\begin{array}{l}\text { Evolución cronológica de } \\
\text { las instituciones }\end{array}$ \\
\hline $\begin{array}{l}\text { Entre palabras de } \\
\text { contenido (del } \\
\text { abstract, del } \\
\text { título, palabras } \\
\text { clave, etc.) }\end{array}$ & $\begin{array}{l}\text { Detección de clusters, } \\
\text { términos relacionados y } \\
\text { líneas de investigación }\end{array}$ \\
\hline $\begin{array}{l}\text { Entre palabras y } \\
\text { códigos de } \\
\text { clasificación }\end{array}$ & $\begin{array}{l}\text { Área de especialización } \\
\text { de las organizaciones }\end{array}$ \\
\hline
\end{tabular}

Figura 16

\section{Difusión de la información y resultados obte- nidos}

El último proceso de la VT es la difusión selectiva de la información generada en función de las necesidades de información del usuario y reflejadas en su perfil. Normalmente el método utilizado para difundirla será el correo electrónico.

\begin{tabular}{|c|c|}
\hline \multicolumn{2}{|c|}{ Software para minería de datos } \\
\hline $\begin{array}{l}\text { Matheo } \\
\text { Analyzer }\end{array}$ & $\begin{array}{l}\text { http://www. matheo- } \\
\text { software.com } \\
\text { Hace estudios bibliométricos y } \\
\text { mapas tecnológicos. } \\
\text { La información se importa de } \\
\text { bases de datos estructuradas. } \\
\text { Permite análisis mediante } \\
\text { recuentos simples y } \\
\text { coocurrencias. } \\
\text { El software es gratis pero hay } \\
\text { que pagar la descarga de } \\
\text { información. }\end{array}$ \\
\hline Tetralogie* & $\begin{array}{l}\text { http://atlas.irit.fr } \\
\text { Es un paquete muy completo } \\
\text { especializado en analizar } \\
\text { grandes volúmenes de } \\
\text { información científica y de } \\
\text { patentes. Su función más } \\
\text { avanzada es la generación de } \\
\text { mapas tecnológicos a partir de } \\
\text { análisis estadísticos de } \\
\text { concurrencias de términos }\end{array}$ \\
\hline \multicolumn{2}{|c|}{ Ambos son de pago } \\
\hline
\end{tabular}

Figura 17 
Como hito final en nuestra cadena de procesos están los resultados que se obtienen, siendo el principal el conocimiento adquirido por la organización para poder anticiparse a los cambios con un menor riesgo en la toma de decisiones.

\section{Conclusiones}

Toda organización que se dedique a la gestión de la I+D+i deberá implantar su sistema de VT en base a lo expresado por la norma UNE 166006:2006 EX-Gestión de la $I+D+i$ : sistemas de vigilancia tecnológica, que clarifica y consolida los servicios relacionados con la VT.

Existen softwares que permiten automatizar de alguna manera los procesos en los que la norma divide la realización de la vigilancia (captar, clasificar, valorizar y distribuir) y es de prever que su número vaya en aumento debido a la más que probable demanda por parte de las organizaciones.

La correcta implantación de un sistema de VT dentro de las organizaciones innovadoras permitirá a éstas:

-Definir las líneas de I+D para las que la empresa tiene buenos recursos propios y para seleccionar socios tecnológicos.

- Mejorar en la selección y comparación de tecnologías para realizar una inversión. cencias.

-Elegir de forma óptima en la negociación de li-

\section{Bibliografía}

Arroyo, Ana; Martínez, Esther. "La vigilancia tecnológica como fuente de generación del conocimiento". En: Robotiker, 2006, n. 7. Consultado en: 03-07-06.

http://revista.robotiker.com/articulos/articulo42/paginal.jsp

Documentos Cotec sobre oportunidades tecnológicas. Vigilancia tecnológica, 14. Consultado: 15-07-06.

http://www.cotec.es/docs/ficheros/200505160025_6_0.pdf

Bergman, M. K. The deep web: surfacing hiden value. Consultado en: 10-07-06.

http://www.brightplanet.com/resources/details/the-deep-websurfacing-hidden-value.html

Callon, Michel; Courtial, Jean Pierre; Penan, Hervé. La scientométrie. Paris: PUF, 1993.

Escorsa, Pere. "De la vigilancia tecnológica a la inteligencia competitiva". En: Conferencia inaugural de los estudios de información y documentación de la UOC del segundo semestre del curso 2001-2002, 2001. Consultado: 10-07-06.

http://www.uoc.edu/web/esp/art/uoc/escorsa0202/escorsa0202.html

Escorsa, Pere; Maspons, Ramon. De la vigilancia tecnológica a la inteligencia competitiva. Madrid: Pearson Educación, 2001, Isbn 84-205-3057-3.

Escorsa, Pere; Maspons, Ramon; Cruz, Elicet. Inteligencia competitiva y transferencia de tecnologías: reflexiones para el desarrollo de la universidad-empresa (ES.3.194). Consultado en: 05-06-06.

http://www.campus-oei.org/salactsi/pere2.pdf
Escorsa, Pere; Valls, Jaume. Tecnología e innovación en la empresa. Barcelona: Edicions UPC, 2003. Isbn 84-8301-706-7.

Fundación madri+d para el Conocimiento. Comunidad virtual de vigilancia tecnológica. Consultado en: 04-06-06.

http://www.madrimasd.org/vt/default.aspx

Giménez, Elea; Román, Adelaida. "Vigilancia tecnológica e inteligencia competitiva: conceptos, profesionales, servicios y fuentes de información". En: El profesional de la información, 2001, mayo, v. 10, n. 5, pp. 11-20

Grupo CDE. Centro de Vigilancia, Normas y Patentes. Las respuestas a todas las preguntas sobre vigilancia tecnológica. Navactiva: el portal para las empresas de Navarra. Vigilancia tecnológica. 01 documentación. Consultado en: 12-07-06.

http://navactiva.com/redirect.jsp? $o=8852$

Hidalgo Nuchera, Antonio. "Vigilancia tecnológica: su importancia estratégica en la empresa". En: Revista Madri+d, 1998, n. 0. Consultado en: 27-01-06.

http://www.madrimasd.org/informacionIDI/revistas/Numero0/debate1.htm

IALE Tecnología. Guía de vigilancia tecnológica: sistema de información estratégica en las pymes. Bilbao: DZ Centro de Diseño, 2003, Isbn 84-7752-348-7. Consultado: 28-06-06.

http://www.zaintek.net/ebizkaia/servlet/EbizkaiaServlet? $P=g L D B \& \# 17$

Maspons, Ramon. "Los sistemas de VT e IC y sus ventajas para las pymes". En: Jornada sobre vigilancia tecnológica e inteligencia competitiva para las pymes de Bizkaia, 2002. Consultado en: 05-07-06.

http://www.zaintek.net/w2/debates/debate_3/indice.html

Norma 166000:2006. Gestión de la $I+D+i$ : terminología y definiciones de las actividades de $I+D+i$. Madrid: Aenor, 2006.

Norma 166001:2006. Gestión de la $I+D+i$ : requisitos de un proyecto de $I+D+i$. Madrid: Aenor, 2006

Norma 166002:2006. Gestión de la I+D+i: requisitos del sistema de gestión de $I+D+i$. Madrid: Aenor, 2006.

Norma UNE 166006 EX. Gestión de la I+D+i: sistema de vigilancia tecnológica. Madrid: Aenor, 2006.

Palop, Fernando; Vicente, José M. Vigilancia tecnológica e inteligencia competitiva: su potencial para la empresa española. Fundación Cotec, 1999. Consultado en: 24-06-06.

http://www.up.univ-mrs.fr/ wlaglea/unapec/intvci/documents/estudio15.pdf

Rodríguez, Marisela. "Análisis de patentes en la inteligencia competitiva y tecnológica: el caso de los materiales avanzados". En: Puzzle, 2003, noviembre-diciembre, n. 8, pp. 4-9. Consultado en: 30-06-06.

http://www.revista-puzzle.com/puzzle_num_2003.htm

Rodríguez, Marisela; Escorsa, Pere. "La inteligencia tecnológica en la organización empresarial: instrumento para la toma de decisiones". En: Revista de la Escuela de Economía y Negocios, 2000, n. 4, pp. 113-142.

Surís, José María; Solé, Francesc; Escorsa, Pere. "Nuevas tecnologías e innovación en las empresas españolas". En: Papeles y memorias de la Real Academia de Ciencias Morales y Políticas, 2001, n. 9, pp. 102-125.

Tecnociencia. Vigilancia tecnológica e inteligencia competitiva. Consultado en: 22-06-06.

http://www.tecnociencia.es/especiales/vigilancia_tecnologica/

Valls, Jaume; Escorsa, Pere. "La innovación y la empresa". En: Dyna, 2000 , v. 75 , n. 8 , pp. 39-46.

Javier Muñoz Durán, Fundación Imabis, Avda. Carlos Haya, 25. 29010 Málaga.

javier.munoz@fundacionimabis.org

María Marín Martínez, Fundación Imabis, Avda. Carlos Haya, 25. 29010 Málaga. maria.marin@fundacionimabis.org

José Vallejo Triano, Hospital Regional Universitario Carlos Haya, Avda. Carlos Haya, s/n. 29010 Málaga jose.vallejo.sspa@juntadeandalucia.es 